\section{Quantified hemodynamics of compression garments}

\author{
Dean J. Bender, ${ }^{1}$ Helane Fronek, ${ }^{2}$ \\ Ed Arkans ${ }^{3}$ \\ ${ }^{1}$ CircAid Medical, San Diego, CA; \\ 'LaJolla Vein, LaJolla, CA; \\ ${ }^{3} \mathrm{ACI}$ Medical, San Marcos, CA, USA
}

\section{Abstract}

Various forms of compression therapy have been utilized for centuries in the treatment of venous disease, with inelastic bandage systems being used in the more acute treatment of severe venous disease and elastic compression stockings used for long-term management of the disease. However, with the advancement in inelastic adjustable compression wraps, we now have the option to consider long-term management of venous disease with an inelastic system and not just elastic systems. The aim of this study was to compare the hemodynamic effect of elastic compression stockings and inelastic compression wraps on venous disease patients when both products are applied to provide the same level of compression. Utilizing the APG device (ACI Medical, San Marcos, CA, USA), venous volumes, venous filling indexes and ejection fraction measurements were captured on 10 patients with varying degrees of venous disease. Measurements were obtained for each patient at baseline (without compression), with either $30-40$ or $20-30 \mathrm{mmHg}$ elastic compression stockings (ECS) and an inelastic compression wrap (ICW) (Juxta-CURES ${ }^{\text {tm }}$ by Circaid Medical, San Diego, CA, USA). The compression level of the ECS was measured at the B1 point utilizing a Picopress ${ }^{\circledR}$ [Microlab Elettronica Sas, Roncaglia di Ponte San Nicolò (PD), Italy] and the ICW was adjusted to provide the exact compression level as the ECS in order to compare the effects of inelasticity versus elasticity independent of compression differences. As expected, the use of compression therapy significantly improved all measures of hemodynamics although it was found that the ICW (average static stiffness 14.3) further improved the measures over ECS (average static stiffness 2.4). Average venous volumes were reduced over baseline with ECS by $19 \%$ while ICW showed a reduction of $35 \%$. Average venous filling indexes were reduced with ECS by $25 \%$ and $39 \%$ with ICW. The ejection fractions for both devices, ECS and ICW, improved an average of $27 \%$. When applying the same compression level, the stiffness associated with ICW can further improve the venous hemody- namics of venous disease patients over ECS. For certain patients, using ICW could prove to be a significant benefit in the management of their disease.

\section{Introduction}

Compression therapy continues to be the principal approach to the management of venous and lymphatic disease around the world. Even with the significant amount of research that has been conducted demonstrating the benefits of inelastic or short-stretch compression therapy over elastic compression stockings (ECS) ${ }^{1-5}$ remain the dominant technology used in the management of chronic venous insufficiency (CVI). However, one main observation of most of these comparisons is that the compression level achieved with inelastic bandaging is significantly higher that that achieved with elastic compression stockings. This is due to the inherent characteristic of inelastic bandages to lose compression over time thus requiring an initial high compression level to provide a therapeutic effect. Additionally with bandages there is no reliable method to apply bandages to a known compression level. ${ }^{6}$ However, now with the advancement of inelastic compression wraps (ICW) to provide a reliable method of achieving known levels of compression that can be adjusted over time by the patient to maintain a therapeutic compression level, we can begin to practically consider the benefits of inelastic compression with improved patient compliance and concordance.

Thus, the purpose of this study is to demonstrate the differences in venous hemodynamics that are provided to venous disease patients when ECS and ICW are used eliminating any discrepancy that may arise from variances in actual compression levels applied.

\section{Materials and Methods}

In this study the venous hemodynamic and compression levels of two compression devices were measured on a total of 10 patients $(\mathrm{M} / \mathrm{F}$ $2: 8$; mean age 56.1 years with a standard deviation of 9.2 years). Nine of the 10 patients were clinically evaluated to have venous disease while the $10^{\text {th }}$ patient demonstrated mild lymphedema in her lower leg with no evidence of venous disease (Table 1).

Utilizing air plethysmography (APG device from ACI Medical, San Marcos, CA, USA) baseline venous hemodynamic data was collected for each patient. The measures included venous volume (VV), Venous filling index (VFI) and ejection fraction (EF). These meas-
Correspondence:Dean J. Bender, CircAid Medical Products Inc., 9323 Chesapeake Drive, Suite B2, San Diego, CA 92123, USA.

E-mail: dbender@circaid.com

Key words: compression, inelastic, elastic, hemodynamics, stockings, juxta-cures, CircAid, venous volume, venous filling index, ejection fraction.

Conference presentation: part of this paper was presented at the International Compression Club (ICC) Meeting on Stiffness of Compression Devices, 2012 May 25, Vienna, Austria (http://www.icc-compressionclub.com/).

Received for publication: 1 0ctober 2012.

Revision received: 19 October 2012.

Accepted for publication: 19 0ctober 2012.

This work is licensed under a Creative Commons Attribution 3.0 License (by-nc 3.0).

CC Copyright D.J. Bender et al., 2013

Licensee PAGEPress, Italy

Veins and Lymphatics 2013; 2:e10

doi:10.4081/vl.2013.e10

ures were taken on the leg in which the patient indicated the worse symptomatic condition (R/L - 6:4).

Each patient was then measured and fit with either a knee-high $30-40 \mathrm{mmHg}$ ECS or a 20-30 mmHg ECS. The actual compression level provided by the stocking was captured utilizing a pressure probe [Picopress ${ }^{\circledR}$, Microlab Elettronica Sas, Roncaglia di Ponte San Nicolò (PD), Italy] placed under the garment at the $\mathrm{B} 1$ position while the patient was in the supine position with their leg slightly elevated. The patient was then asked to stand firmly on both feet and a second compression level reading was captured in order to determine the static stiffness index of the ECS (Figure 1). The venous hemodynamic measures were then repeated with the APG device while the ECS remained in place.

The stocking was removed and each patient was then fit with an ICW (Juxta-Cures ${ }^{\mathrm{TM}}$ from CircAid Medical Products, San Diego, CA, USA). The ICW was adjusted to provide the same compression level achieved with the ECW $( \pm 1 \mathrm{mmHg})$ in the supine position and a second compression measured was captured in the standing position. The venous hemodynamic measures were again repeated while the compression wrap remained in place.

\section{Results}

With the compression levels of the ECS and the ICW essentially equivalent for each patient, we were able to determine the Static 
Stiffness Index exerted by each compression device. Static Stiffness of a compression device is defined as the difference between the compression exerted at the B1 point in the standing position versus the supine. The results (Figure 2) clearly demonstrate that the ECS provided a low static stiffness index with an average of $2.4 \mathrm{mmHg}$, while the ICW produced an average static stiffness of 14.3 $\mathrm{mmHg}$.

The results from the APG measurements were as expected with both the ECS and the ICW significantly improving all three measures over baseline. Furthermore, it was found that the ICW provided a significant improvement over the ECS in VV and VFI reduction.

The ECS reduced the VV (Figure 3) by an average of $19 \%$ (baseline avg - $135.5 \mathrm{~mL}$ and ECS avg - $109.0 \mathrm{~mL}$ ). The ICW reduced VV by an average of $35 \%$ (ICW avg - $86.4 \mathrm{~mL}$ ).

Similar reductions were seen in the VFI (Figure 4) with a baseline avg - $2.9 \mathrm{~mL} / \mathrm{s}$; ECS avg $-2.2 \mathrm{~mL} / \mathrm{s}$ (25\% reduction from baseline) and the ICW avg $-1.7 \mathrm{~mL} / \mathrm{s}$ (39\% reduction from baseline).

EF (Figure 5) for both compression devices significantly improved over baseline with both devices averaging an improvement of $27 \%$.

\section{Discussion and Conclusions}

The effect of compression devices on the venous system depends on two key factors; the pressure exerted on the limb and the stiffness of the materials used in the device. ECS devices are typically elastic in nature and are designed to provide a given compression range (mmHg) in the ankle region as defined by the manufacturer (i.e. $30-40 \mathrm{mmHg}$ ). Because of the high elasticity in ECS devices the resulting fabric is not stiff and as such stretches with the movements of the limb. ECS devices can be thought to provide static stiffness where the compression level provided is essentially unchanged as the user moves from supine to standing to walking positions. ICW have been available for over 20 years and deliver a compression level that is dependent upon the amount of tension applied to the closing straps. Not until the past few years has such a device been able to deliver a known level of compression similar to the of ECS devices. This has been achieved by the inclusion of a Built-in Pressure System, BPSTM (CircAid Medical Products), which correlates the tension applied to the closing straps and the circumference of the limb to a known pressure range. As the name indicates, ICWs are inelastic, stiff in nature. This inelasticity has been demonstrated to provide a dynamic compression under the device where the compression level increases and decreases dramatically as the patient moves from supine to standing to walking positions.

Because ECS devices are readily available, have known compression levels, are aesthetically pleasing and relatively easy to apply for a patient when compared to bandaging, they have become the dominant technology in the treatment and management of CVI around the world. However, now that ICW devices are becoming more prominent, have known compression levels and are easy for the patient to apply, we have the opportunity to consider the effect of stiffness (dynamic compression) in our treatment of CVI.

This study was designed to eliminate the variable of compression level from the assess- ment of the effectiveness of the device by applying equal compression levels at the B1 point. This was achieved by adjusting the ICW straps until a near equivalent compression reading was obtained on the pressure monitor. By eliminating the compression variable we are able to compare the effect that stiffness of the compression device exerts on any given patient.

Our results clearly showed that the ICW was stiff and delivered a higher working pressure (14.3 $\mathrm{mmHg}$ ) when the patients were in the standing position versus supine, while the ECS $(2.4 \mathrm{mmHg})$ resulted in little to no increase in pressure on the same patients.

As expected, both compression devices significantly improved the patient's venous hemodynamics. Applying pressure to the tissue of the limb and thus preventing the expansion of the veins during refilling maintains a smaller total volume of the complete venous system. However, due to the inelastic nature of the ICW and the fact that the device has limited stretch under movement, the reduction in $\mathrm{VV}$ was significantly greater for the ICW $(36 \% \mathrm{P}=0.008)$ than that achieved with the ECS (20\% $\mathrm{P}=0.009$ ). Similarly, the inelasticity of the ICW resulted in a $40 \%(\mathrm{P}=0.028)$ reduction in VFI versus $23 \%$ ( $\mathrm{P}=0.009)$ for the $\mathrm{ECS}$, compared to baseline measurements without a compression device.

Interestingly, both ECS and ICW improved the EF by $27 \%$ on average, although the measures did not achieve statistical significance (ICW $\mathrm{P}=0.110$; ECS $\mathrm{P}=0.055$ ). The results on average were contrary to our expectations in that Mosti and Partsch ${ }^{7}$ reported in 2010 higher EF percentages with inelastic bandages versus ECS when measuring with strain-gauge plethsmography, although 7 out of the 10

Table 1. Patient population.

$\begin{array}{lcccccc}\begin{array}{l}\text { Patient } \\ \text { no. }\end{array} & \text { CEAP } & \text { Gender } & \text { Age } & \begin{array}{c}\text { Limb } \\ \text { Stocking } \\ \text { label } \\ \text { compression }\end{array} & \begin{array}{c}\text { Pressure } \\ \text { B1 }\end{array} \\ 1 & \text { (mm Hg) }\end{array}$

\section{Compression levels}

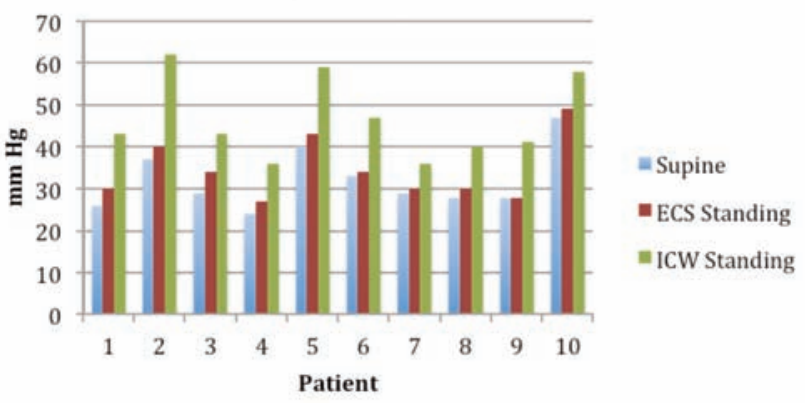

Figure 1. Compression levels measured at the B1 position. ECS elastic compression stockings; ICW, inelastic compression wrap. 
Static stiffness index

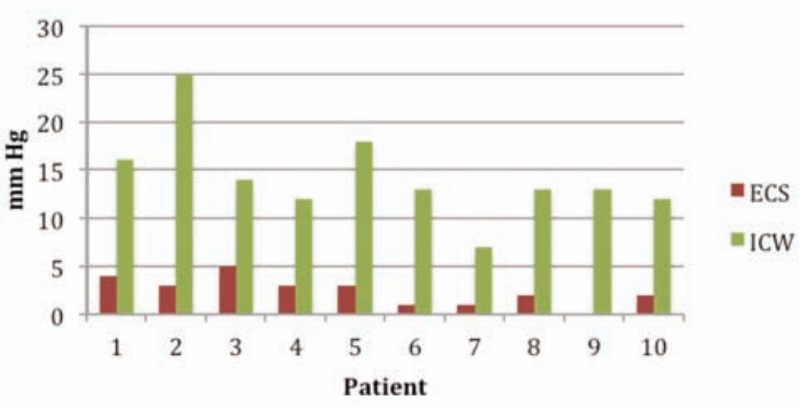

Figure 2. Static stiffness index (compression level difference between standing and supine at the $\mathrm{B} 1$ position measured in $\mathrm{mmHg}$ ). ECS, elastic compression stockings; ICW, inelastic compression wrap.

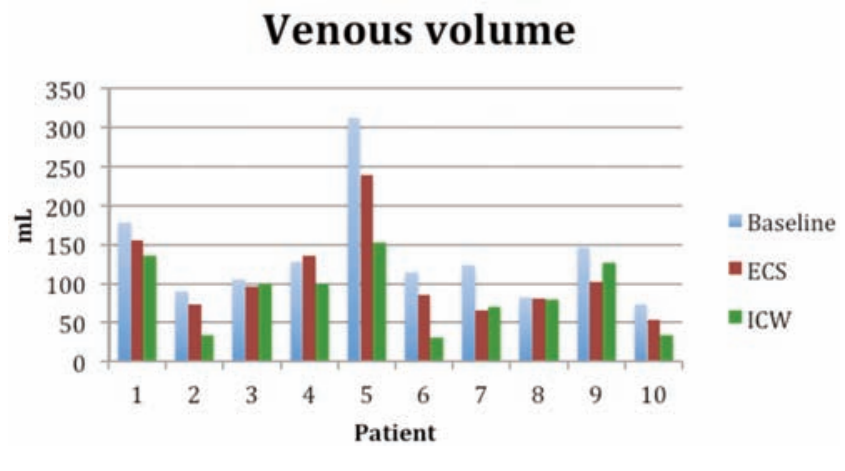

Figure 3. Total venous volume. ECS, elastic compression stockings; ICW, inelastic compression wrap.

\section{Venous filing index}

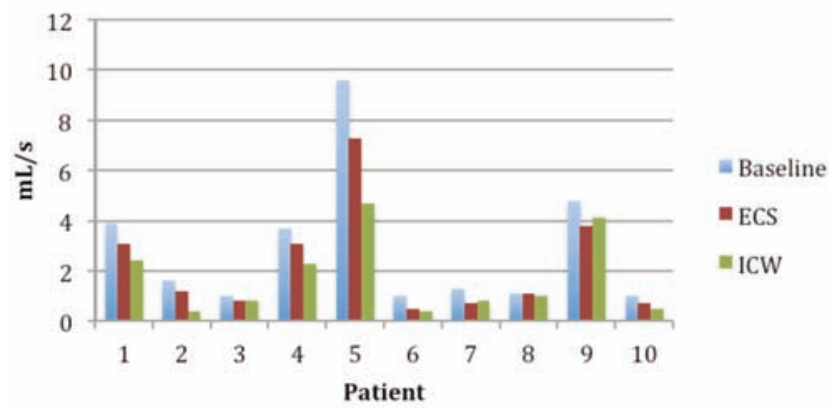

Figure 4. Venous filling index - rate of venous refilling. ECS, elastic compression stockings; ICW, inelastic compression wrap.

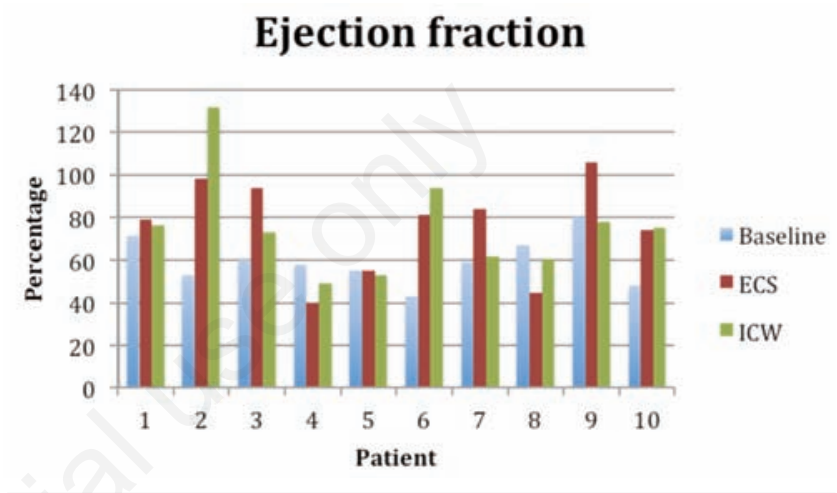

Figure 5. Ejection fraction - percentage of venous blood expelled as a result of a single calf flex. ECS, elastic compression stockings; ICW, inelastic compression wrap. patients did see an improvement in EF with the ICW over ECS.

When considering this outcome further in view of our assumption that the EF with the ICW would be significantly improved versus the ECS, we observed on a patient-by-patient basis for all 3 variables measured (VV, VFI and EF) that patients number 3,7 and 9 (Figure 5) had equivalent or superior results with the ECS versus the ICW. This suggests that there was something unique about these patients that allowed the ECS to perform better than the ICW in spite of the greater elasticity. Unfortunately, we did not observe anatomical characteristics of these patients in order to determine if a correlation exists between anatomy of the limb and the effect of compression garments. One theory is that certain tissue characteristics may be influenced more by the tension applied by an ECS once stretched, resulting in an increased force inward on the limb. In contrast, the lack of elasticity of the ICW simply prevents the limb from expanding, but does not reduce limb size based on movement. Another thought is that the tissue make-up defuses the compression differently, thus mitigating the expected effect of the inelastic device.
Regardless, this is a phenomenon that we believe justifies further investigation and recommend that additional work be conducted to determine what variables should be considered in regards to determining when an elastic device should be chosen over an inelastic device. It is our intention to repeat this study including an anatomical and ultrasound evaluation of each patient and to also monitor sub-garment compression levels throughout the various tests.

In conclusion, this study confirms that inelastic compression devices provide a superior hemodynamic effect on average and should be considered when the disease state dictates the need for the maximum impact on the circulatory system.

\section{References}

1. Kline $\mathrm{CN}$, Macias BR, Kraus E, et al. Inelastic compression legging produces gradient compression and significantly higher skin surface pressures compared with an elastic compression stocking. Vascular
2008;16:25-30.

2. Spence RK, Cahall E. Inelastic versus elastic leg compression in chronic venous insufficiency: a comparison of limb size and venous hemodynamics. J Vasc Surg 1996; 24:783-7.

3. Callam MJ, Harper DR, Dale JJ, et al. Lothian and forth valley leg ulcer healing trial. 1. elastic versus nonelastic bandaging in the treatment of chronic leg ulceration. Phlebology 1992;7:136-41.

4. Charles H. Compression healing of ulcers. J District Nurs 1991;4:6-7.

5. Partsch H, Horakova MA. Compression stockings for the treatment of venous leg ulcers [Kompressionstrumpfe zur Behandlung venoser Unterschenkelgeschwure]. WienerMedizineWochenschrift 1994;144:242-9.

6. Lurie F, Kistner R. Interface pressure under compression bandages: Current practice and a way to consistency. Poster Presentation American Venous Forum, February 9th 2012, Orlando, FL, USA.

7. Mosti G, Partsch H. Measuring venous pumping function by strain-gauge plethysmography. Int Angiol 2010;29:421-5. 